\title{
Diffusion and Elastic Equations on Networks
}

\author{
By \\ Soon-Yeong Chung, Yun-Sung ChUnG** and Jong-Ho KIM***
}

\begin{abstract}
In this paper, we discuss discrete versions of the heat equations and the wave equations, which are called the $\omega$-diffusion equations and the $\omega$-elastic equations on graphs. After deriving some basic properties, we solve the $\omega$-diffusion equations under (i) the condition that there is no boundary, (ii) the initial condition and (iii) the Dirichlet boundary condition. We also give some additional interesting properties on the $\omega$-diffusion equations, such as the minimum and maximum principles, Huygens property and uniqueness via energy methods. Analogues of the $\omega$-elastic equations on graphs are also discussed.
\end{abstract}

\section{$\S 1$. Introduction}

Today network structures can be found in almost every fields of our life. For example, the brain is a network of neurons; organizations are people networks; the global economy, food webs, molecules, and the internet can all be represented as networks. Since network represents a structure of every materials interconnecting any pair of users or nodes by means of some meaningful links, it is quite natural that its structure can be represented by a connected graph whose vertices represent nodes and whose edges represent their links.

Communicated by H. Okamoto. Received November 10, 2005. Revised May 11, 2006. 2000 Mathematics Subject Classification(s): Primary 05C40, 35R30; Secondary 94C12. Key words: discrete Laplacian, diffusion kernel, elastic kernel.

The first author was supported by Korea Research Foundation Grant (KRF-2003-041C00023) and Sogang University in 2004.

The second author is supported by BK21 Project.

* Department of Mathematics and Program of Integrated Biotechnology, Sogang University, Seoul 121-742, Korea.

**BK21 Math Modeling HRD Div., Department of Mathematics, Sungkyunkwan University, Suwon 440-746, Korea.

***National Institute for Mathematical Sciences, Daejeon, 305-340, Korea. 
Among the various problems on networks, solving direct and inverse problems of an equation, called $\omega$-Laplace equation on graphs which can be interpreted as a diffusion equation on electric networks has been studied by a number of authors such as $[\mathrm{B}],[\mathrm{CvDS}],[\mathrm{CY}],[\mathrm{MC}]$ and so on.

Recently, in the paper [CB], the first author and C. A. Berenstein introduced another method-PDE on graphs-to study problems of the $\omega$-Laplace equation on graphs. They defined discrete analogues of some notions on calculus such as integration, directional derivative, gradient and so on and then showed that some fundamental properties on calculus, for example Green's theorem, are nicely behaved on weighted graphs. By using these properties, they proved the solvability of direct problems such as the Dirichlet and Neumann boundary value problems of the $\omega$-Laplace equation on graphs. Moreover, they showed the global uniqueness of the inverse problem of the equation under the monotonicity condition.

In this paper, by using the notions in $[\mathrm{CB}]$, we discuss the $\omega$-diffusion equations and the $\omega$-elastic equations on graphs, which can be used in various areas, for example, modelling of energy flows through a network or modelling of vibration of molecules, and so on. Our main concern in this paper is to solve $\omega$-diffusion equations and $\omega$-elastic equations for the cases that (i) there is no boundary, (ii) an initial condition is given and (iii) a Dirichlet boundary condition is given. To represent their solutions, we use the $\omega$-diffusion kernel and the $\omega$-elastic kernel, which are constructed by eigenfunctions of the $\omega$-Laplacian operator. There have been some works on the $\omega$-diffusion equations and the $\omega$-elastic equations on graphs (for example, see [Ch] and [CvDS]), however finding solutions to their initial and boundary problems and representing them by means of their kernels have not been studied so far precisely in the literature, as far as the authors know.

Besides, we offer some additional interesting properties on the $\omega$-diffusion equations and the $\omega$-elastic equations on graphs such as the minimum and maximum property, the Huygens property and proving uniqueness of solutions via energy method.

Remark. There have been some papers, for example [CY], which deal with the $\omega$-diffusion kernel, however the $\omega$-elastic kernel has not been introduced, as far as the authors know. 


\section{§2. Preliminaries}

We start with graph theoretic notions frequently used throughout this paper. By a graph $G=G(V, E)$ we mean a finite set $V$ of vertices with a set $E$ of two-element subsets of $V$ (whose elements are called edges). As conventionally used, we denote either $x \in V$ or $x \in G$ the fact that $x$ is a vertex in $\mathrm{G}$.

A graph $G$ is said to be simple if it has neither multiple edges nor loops, and $\mathrm{G}$ is said to be connected if for every pair of vertices $x$ and $y$ there exist a sequence of vertices $x=x_{0} \sim x_{1} \sim x_{2} \sim \cdots \sim x_{n-1} \sim x_{n}=y$ such that $x_{j-1}$ and $x_{j}$ are connected by an edge (termed adjacent) for $j=1,2, \cdots, n$, where $x \sim y$ means that two vertices $x$ and $y$ are connected (adjacent) by an edge in $E$.

A graph $S=S\left(V^{\prime}, E^{\prime}\right)$ is said to be a subgraph of $G(V, E)$ if $V^{\prime} \subset V$ and $E^{\prime} \subset E$. Then, we call $\mathrm{G}$ a host graph of S. If $E^{\prime}$ consists of all the edges from $E$ which connect the vertices of $V^{\prime}$ in its host graph $G$, then $S$ is called an induced subgraph. A weighted (undirected) graph is a graph $G(V, E)$ associated with a weight function $\omega: V \times V \rightarrow[0, \infty)$ satisfying

(i) $\omega(x, y)=\omega(y, x), \quad x, y \in V$

(ii) $\omega(x, y)=0$ if and only if $\{x, y\} \notin E$.

Here, $\{x, y\}$ denotes the edge connecting the vertices $x$ and $y$. The degree $d_{\omega} x$ of a vertex $x$ is defined to be

$$
d_{\omega} x:=\sum_{y \in V} \omega(x, y)
$$

Throughout this paper, all the subgraphs in our concern are assumed to be induced, simple and connected subgraphs of a weighted graph. A function on a graph is understood to be a function defined just on the set of vertices.

The integration of a function $f: G \rightarrow \mathbf{R}$ on a graph $G=G(V, E)$ is defined by

$$
\int_{G} f d_{\omega} \quad\left(\text { or simply } \int_{G} f\right):=\sum_{x \in V} f(x) d_{\omega} x .
$$

The volume of a graph $G$ is defined to be $\operatorname{vol}(G):=\int_{G} d_{\omega} x=\sum_{x \in V} d_{\omega} x$.

For the directional derivative of a function $f: G \rightarrow \mathbf{R}$, we mean

$$
D_{\omega, y} f(x):=[f(y)-f(x)] \sqrt{\frac{\omega(x, y)}{d_{\omega} x}}, \quad x, y \in V
$$


and the gradient $\nabla_{\omega}$ of a function $f$ is defined to be a vector

$$
\nabla_{\omega} f(x):=\left(D_{\omega, y} f(x)\right)_{y \in V},
$$

which is indexed by the vertices $y \in V$. For a subgraph $S$ of a graph $G=$ $G(V, E)$, the (vertex) boundary $\partial S$ of $S$ is the set of all vertices $z \in V$ not in $S$ but adjacent to some vertex in $S$, i.e.

$$
\partial S:=\{z \in V \backslash S \mid z \sim y \text { for some } y \in S\} .
$$

Also, by $\bar{S}$ we denote a graph whose vertices and edges are in $S$ and vertices in $\partial S$.

The (outward) normal derivative $\frac{\partial f}{\partial_{\omega} n}(z)$ at $z \in \partial S$ is defined to be

$$
\frac{\partial f}{\partial_{\omega} n}(z):=\sum_{y \in S}[f(z)-f(y)] \cdot \frac{\omega(z, y)}{d_{\omega}^{\prime} z},
$$

where $d_{\omega}^{\prime} z=\sum_{y \in S} \omega(z, y)$.

The $\omega$-Laplacian $\Delta_{\omega}$ of a function $f: G \rightarrow \mathbf{R}$ on a graph $G$ is defined by

$$
\begin{aligned}
\Delta_{\omega} f(x): & =-\sum_{y \in V} D_{\omega, y}\left(D_{\omega, y} f(x)\right) \\
& =\sum_{y \in V}[f(y)-f(x)] \cdot \frac{\omega(x, y)}{d_{\omega} x}, \quad x \in V .
\end{aligned}
$$

For more details, we refer to $[\mathrm{CB}],[\mathrm{Ch}]$ and $[\mathrm{CvDS}]$.

In what follows, a function $f$ defined on $\bar{S}$ may be understood as a function on its host graph $G$ such that $f=0$ on $G \backslash \bar{S}$, if necessary.

The following theorem was proved by the first author and C. A. Berenstein in the paper $[\mathrm{CB}]$.

Theorem 2.1 [CB]. Let $S$ be a subgraph of a host graph $G$. Then for any pair of functions $f: \bar{S} \rightarrow \mathbf{R}$ and $h: \bar{S} \rightarrow \mathbf{R}$, we have

$$
2 \int_{\bar{S}} h\left(-\Delta_{\omega} f\right)=\int_{\bar{S}} \nabla_{\omega} h \cdot \nabla_{\omega} f
$$

(ii)

$$
2 \int_{\bar{S}} f\left(-\Delta_{\omega} f\right)=\int_{\bar{S}}\left|\nabla_{\omega} f\right|^{2} .
$$


(iii)

$$
\int_{\bar{S}} h \Delta_{\omega} f=\int_{\bar{S}} f \Delta_{\omega} h
$$

(iv) (Green's formula)

$$
\int_{S}\left(f \Delta_{\omega} h-h \Delta_{\omega} f\right)=\int_{\partial S}\left(f \frac{\partial h}{\partial_{\omega} n}-h \frac{\partial f}{\partial_{\omega} n}\right) .
$$

Properties in the Theorem 2.1 are very interesting in a sense that some notations in this paper, such as integration, directional derivatives, Laplacian, gradient and normal derivative behaves very similar to those in classical vector calculus. This similarity enables us to study partial differential equations on weighted graphs.

\section{$\S 3 . \quad \omega-D i f f u s i o n$ Equations on Weighted Graphs}

This section deals with properties of solutions $F(x, t)$ of the following equations on a graph $G(V, E)$ with a weight $\omega$,

$$
\partial_{t} F(x, t)-\Delta_{\omega} F(x, t)=H(x, t), \quad x \in V, \quad t \in(0, T),
$$

where $H(x, t)$ is a given function in $V \times(0, T)$ with $T$ a given positive real number or $\infty$. (Throughout this paper, we always denote $T$ to be a given positive real number or $\infty$.) The equation (3.1) is said to be the $\omega$-diffusion equations on the graph $G$. Especially, for the case of $H(x) \equiv 0$, we say the equation is homogeneous.

We start with giving a physical interpretation of the $\omega$-diffusion equation. Let a graph $G=G(V, E)$ and a weight $\omega$ be given. Consider a function $F$ : $V \times[0, T) \rightarrow \mathbf{R}$, where $F(x, t)$ represents the potential of energy given at each vertex $x \in V$ at time $t \in[0, T)$. Assume that the energy flows from a vertex $x$ to its adjacent vertex $y$ through edge. If we give an assumption (which is very natural in many situations) that the rate of change of the quantity flows from $x$ to $y$ is proportional to (i) the difference of the quantity of the material of two vertices $x$ and $y$ and (ii) the conductivity $\omega(x, y)$ of the edge between $x$ and $y$, then it is easy to see that the function $F$ satisfy the equation

$$
\partial_{t} F(x, t)=\sum_{y \in V}[F(y, t)-F(x, t)] \frac{\omega(x, y)}{d_{\omega} x}, \quad x \in V, t \in[0, T),
$$

which is the homogeneous $\omega$-diffusion equation. 
In what follows, for an interval $I \in \mathbf{R}$, we say that a function $f: V \times I \rightarrow \mathbf{R}$ belongs to $\mathcal{C}^{n}(V \times I)$ if for each $x \in V$, the function $f(x, \cdot)$ is $n$-times differentiable in $I$ and $\left(\frac{d}{d t}\right)^{n} f(x, \cdot)$ is continuous in $I$. We use the notation $L^{1}(V \times I)$ to denote the set of functions $f: V \times I \rightarrow \mathbf{R}$ such that for each $x \in V, f(x, \cdot)$ is integrable on $I$.

The first property we are to investigate is the minimum and maximum principles.

Theorem 3.1. $\quad$ Let $S$ be a subgraph of a host graph $G$ with a weight $\omega$. If a function $F \in \mathcal{C}^{0}(\bar{S} \times[0, T))$ satisfies

$$
\left\{\begin{array}{l}
\partial_{t} F(x, t)-\Delta_{\omega} F(x, t) \geq 0, \quad x \in S, t \in(0, T) \\
F(z, t) \geq 0, \quad z \in \partial S, \quad t \in[0, T) \\
F(x, 0) \geq 0, \quad x \in S
\end{array}\right.
$$

then we have

$$
F(x, t) \geq 0, \quad x \in \bar{S}, t \in[0, T]
$$

Proof. Let $T_{0} \in(0, T)$. For an arbitrary $\epsilon>0$, define $H: \bar{S} \times\left[0, T_{0}\right] \rightarrow \mathbf{R}$ as $H(x, t):=F(x, t)+\epsilon t$. Then since $H(x, \cdot)$ is continuous on $\left[0, T_{0}\right]$ for all $x \in \bar{S}, H$ has a minimum value at $\left(x_{0}, t_{0}\right) \in \bar{S} \times\left[0, T_{0}\right]$. We show that $x_{0} \in \partial S$ or $t_{0}=0$. Suppose on the contrary that $x_{0} \in S$ and $t_{0} \in\left(0, T_{0}\right]$. Then by elementary calculus, we see that

$$
\partial_{t} H\left(x_{0}, t_{0}\right) \leq 0 \quad\left(\partial_{t} H\left(x_{0}, t_{0}\right)=0, \quad \text { if } \mathrm{t}_{0}<\mathrm{T}_{0}\right)
$$

and

$$
\Delta_{\omega} H\left(x_{0}, t_{0}\right)=\sum_{y \in \bar{S}}\left[H\left(y, t_{0}\right)-H\left(x_{0}, t_{0}\right)\right] \frac{w\left(y, x_{0}\right)}{d_{\omega} x_{0}} \geq 0
$$

which implies

$$
\partial_{t} H\left(x_{0}, t_{0}\right)-\Delta_{\omega} H\left(x_{0}, t_{0}\right) \leq 0
$$

But we also have

$$
\partial_{t} H\left(x_{0}, t_{0}\right)-\Delta_{\omega} H\left(x_{0}, t_{0}\right)=\left[\partial_{t} F\left(x_{0}, t_{0}\right)-\Delta_{\omega} F\left(x_{0}, t_{0}\right)\right]+\epsilon>0,
$$

which is a contradiction. Hence it should be true that

$$
x_{0} \in \partial S \quad \text { or } \quad \mathrm{t}_{0}=0 \text {, }
$$


which implies that $F\left(x_{0}, t_{0}\right) \geq 0$ by the hypothesis. Consequently, if $(x, t)$ is any point in $\bar{S} \times\left[0, T_{0}\right]$, then

$$
\begin{aligned}
F(x, t) & =H(x, t)-\epsilon t \geq H\left(x_{0}, t_{0}\right)-\epsilon t \\
& =F\left(x_{0}, t_{0}\right)+\epsilon t_{0}-\epsilon t \\
& \geq \epsilon\left(t_{0}-t\right) .
\end{aligned}
$$

Since $\epsilon>0$ is arbitrary, the inequality implies that $F(x, t) \geq 0,(x, t) \in \bar{S} \times$ $\left[0, T_{0}\right]$. Since $T_{0} \in(0, T)$ can be chosen arbitrary, we get the result.

The following corollaries are immediate consequences of the previous theorem:

Corollary 3.1. $\quad$ Let $S$ be a subgraph of a host graph $G$ with a weight $\omega$. If a function $F \in \mathcal{C}^{0}(\bar{S} \times[0, T))$ satisfies

$$
\left\{\begin{array}{l}
\partial_{t} F(x, t)-\Delta_{\omega} F(x, t) \leq 0, \quad x \in S, t \in(0, T) \\
F(z, t) \leq 0, \quad z \in \partial S, t \in[0, T) \\
F(x, 0) \leq 0, \quad x \in S
\end{array}\right.
$$

then we have

$$
F(x, t) \leq 0, \quad x \in \bar{S}, t \in[0, T]
$$

Proof. The proof is obvious from the result of the previous theorem, if we consider a function $\widetilde{F}(x, t):=-F(x, t)$.

Corollary 3.2. $\quad$ Let $S$ be a subgraph of a host graph $G$ with a weight $\omega$. If a function $F \in \mathcal{C}^{0}(\bar{S} \times[0, T))$ satisfies

$$
\left\{\begin{array}{l}
\partial_{t} F(x, t)-\Delta_{\omega} F(x, t)=0, \quad x \in S, t \in(0, T) \\
F(z, t)=0, \quad z \in \partial S, \quad t \in[0, T) \\
F(x, 0)=0, \quad x \in S
\end{array}\right.
$$

then we have

$$
F(x, t)=0, \quad x \in \bar{S}, t \in[0, T] .
$$

Proof. It follows easily from Theorem 3.1 and Corollary 3.1.

Remark. Authors borrow the idea of proving the previous theorem and corollaries from the book [W] by D. V. Widder, where the minimum and maximum principles of the classical heat equation was proved. 
For a function $f: G \rightarrow \mathbf{R}$, with $|V|=N$, we may consider it as a $N$ dimensional vector. By the same manner, the $\omega$-Laplacian operator $\Delta_{\omega}$ also can be considered as a matrix defined by

$$
\Delta_{\omega}(x, y)=\left\{\begin{array}{cl}
-1, & \text { if } x=y \\
\frac{\omega(x, y)}{d_{\omega} x}, & \text { if } x \sim y \\
0, & \text { otherwise }
\end{array}\right.
$$

Let $D$ denotes the diagonal matrix with $(x, x)$-th entry having the value $d_{\omega} x$ for each $x$ and $\mathcal{L}_{\omega}=D^{1 / 2} \Delta_{\omega} D^{-1 / 2}$. Then $\left(-\mathcal{L}_{\omega}\right)$ is a nonnegative definite symmetric matrix, so that it has the eigenvalues

$$
0<\lambda_{0} \leq \lambda_{1} \leq \lambda_{2} \leq \ldots \leq \lambda_{N-1},
$$

and corresponding eigenfunctions

$$
\Phi_{0}, \Phi_{1}, \Phi_{2}, \ldots, \Phi_{N-1},
$$

which are orthonormal in the sense that for each pair of distinct $i$ and $j$

$$
\sum_{x \in V} \Phi_{i}(x) \cdot \Phi_{j}(x)=0
$$

while, for all $j$,

$$
\sum_{x \in V}\left|\Phi_{j}(x)\right|^{2}=1
$$

It is easy to show that $\lambda_{0}=0, \lambda_{1}>0$ and $\Phi_{0}(x)=\frac{\sqrt{d_{\omega} x}}{\sqrt{\operatorname{vol}(G)}}, \quad x \in V$. In what follows, we occasionally use the notation $\langle,\rangle_{X}$, defined by $\langle f, g\rangle_{X}=$ $\sum_{x \in X} f(x) g(x)$ for simplicity.

The following theorem characterizes solutions of the $\omega$-diffusion equations on graphs without boundary condition.

Theorem 3.2. Let $G(V, E)$ be a graph with a weight $\omega$ and $H(x, t) \in$ $\mathcal{C}^{0}(V \times(0, T)) \cap L^{1}(V \times(0, T))$. Then every solution $F(x, t)$ of the equation

$$
\partial_{t} F(x, t)-\Delta_{\omega} F(x, t)=H(x, t), \quad x \in V, t \in(0, T)
$$

is represented as follows: there exist $c_{0}, c_{1}, \ldots, c_{N-1}$ such that

$$
\begin{aligned}
& F(x, t) \\
& =\frac{1}{\sqrt{d_{\omega} x}} \sum_{j=0}^{N-1}\left[c_{j}+\int_{0}^{t}\left\langle D^{1 / 2} H(\cdot, \tau), \Phi_{j}\right\rangle_{V} e^{\lambda_{j} \tau} d \tau\right] e^{-\lambda_{j} t} \Phi_{j}(x),
\end{aligned}
$$

for $x \in V$ and $t \in(0, T)$, where $N=|V|$. 
Proof. Consider the expansion

$$
\left(D^{1 / 2} F\right)(x, t)=\sum_{j=0}^{N-1} a_{j}(t) \Phi_{j}(x), \quad x \in V,
$$

where $a_{j}(t)=\left\langle D^{1 / 2} F(\cdot, t), \Phi_{j}\right\rangle, j=0,1,2, \ldots, N-1$. Then since $\mathcal{L}_{\omega} D^{1 / 2}=$ $D^{1 / 2} \Delta_{\omega}$ and

$$
\begin{aligned}
-\lambda_{j} a_{j}(t) & =\left\langle D^{1 / 2} F(\cdot, t), \mathcal{L}_{\omega} \Phi_{j}\right\rangle_{V} \\
& =\left\langle\mathcal{L}_{\omega} D^{1 / 2} F(\cdot, t), \Phi_{j}\right\rangle_{V} \\
& =\left\langle D^{1 / 2}\left[\partial_{t} F(\cdot, t)-H(\cdot, t)\right], \Phi_{j}\right\rangle_{V} \\
& =a_{j}^{\prime}(t)-\left\langle D^{1 / 2} H(\cdot, t), \Phi_{j}\right\rangle_{V},
\end{aligned}
$$

we have

$$
a_{j}(t)=e^{-\lambda_{j} t}\left[c_{j}+\int_{0}^{t} e^{\lambda_{j} \tau} \cdot \sum_{y \in V} H(y, \tau) \Phi_{j}(y) \sqrt{d_{\omega} y} d \tau\right], \quad j=0,1, \ldots, N-1
$$

for some real constants $c_{0}, c_{1}, \ldots, c_{N-1}$. Hence

$$
\sqrt{d_{\omega} x} F(x, t)=\sum_{j=0}^{N-1}\left[c_{j}+\int_{0}^{t} \sum_{y \in V} e^{\lambda_{j} \tau} H(y, \tau) \Phi_{j}(y) \sqrt{d_{\omega} y} d \tau\right] e^{-\lambda_{j} t} \Phi_{j}(x),
$$

equivalently,

(3.4) $F(x, t)$

$$
=\frac{1}{\sqrt{d_{\omega} x}} \sum_{j=0}^{N-1}\left[c_{j}+\int_{0}^{t} \sum_{y \in V} H(y, \tau) \Phi_{j}(y) \sqrt{d_{\omega} y} e^{\lambda_{j} \tau} d \tau\right] e^{-\lambda_{j} t} \Phi_{j}(x) .
$$

Moreover, it is easy to see that $F(x, t)$ in (3.4) satisfies the equation (3.3).

Remark. One can easily see from the solutions (3.3) that the regularity of the solutions depends on the regularity of the function $H(x, t)$. Precisely speaking, if $H(x, t)$ is in $\mathcal{C}^{k}(V \times(0, T))$, then the solutions $F(x, t)$ belong to $\mathcal{C}^{k+1}(V \times(0, T))$.

The following Corollary deals with the homogeneous case of the previous result.

Corollary 3.3. Let $G(V, E)$ be a graph with a weight $\omega$. Every solution $F(x, t)$ of the equation

$$
\partial_{t} F(x, t)-\Delta_{\omega} F(x, t)=0, \quad x \in V, t \in(0, T),
$$


is represented as follows: there exist $c_{0}, c_{1}, \ldots, c_{N-1}$ such that

$$
F(x, t)=\frac{1}{\sqrt{d_{\omega} x}} \sum_{j=0}^{N-1} c_{j} e^{-\lambda_{j} t} \Phi_{j}(x), \quad x \in V, t \in(0, T),
$$

where $N=|V|$.

Proof. The proof follows at once, letting $H(x, t) \equiv 0$ in $(3.3)$.

For a graph $G(V, E)$ with a weight $\omega$, the function $E_{\omega}: V \times V \times[0, T) \rightarrow \mathbf{R}$ defined by

$$
E_{\omega}(x, y, t)=\sum_{j=0}^{|V|-1} e^{-\lambda_{j} t} \Phi_{j}(x) \Phi_{j}(y) \sqrt{\frac{d_{\omega} y}{d_{\omega} x}}, \quad x, y \in V, t \in[0, T),
$$

is called the $\omega$-diffusion kernel.

We now employ the $\omega$-diffusion kernel to represent a solution to the Cauchy problem of the $\omega$-diffusion equation.

Theorem 3.3. Let $G(V, E)$ be a graph with a weight $\omega, H: V \times[0, T) \rightarrow$ $\mathbf{R}$ be a function belong to $\mathcal{C}^{0}(V \times(0, T)) \cap L^{1}(V \times(0, T))$ and $f: V \rightarrow \mathbf{R}$ be given. Then the unique solution $F(x, t) \in \mathcal{C}^{0}(V \times[0, T))$ of the following Cauchy problem

$$
\left\{\begin{array}{l}
\partial_{t} F(x, t)-\Delta_{\omega} F(x, t)=H(x, t), \quad x \in V, t \in(0, T) \\
F(x, 0)=f(x), \quad x \in V
\end{array}\right.
$$

is given by

$$
F(x, t)=\left\langle E_{\omega}(x, \cdot, t), f\right\rangle_{V}+\int_{0}^{t}\left\langle E_{\omega}(x, \cdot, t-\tau), H(\cdot, \tau)\right\rangle_{V} d \tau,
$$

for $x \in V$ and $t \in[0, T)$.

Proof. We first discuss how to construct the solution (3.7) from the equation (3.6). By virtue of Theorem 3.2, if $F(x, t)$ is a solution of the equation (3.6), then it satisfies

(3.8) $F(x, t)$

$$
=\frac{1}{\sqrt{d_{\omega} x}} \sum_{j=0}^{N-1}\left[c_{j}+\sum_{y \in V} \int_{0}^{t} e^{\lambda_{j} \tau} H(y, \tau) d \tau \Phi_{j}(y) \sqrt{d_{\omega} y}\right] e^{-\lambda_{j} t} \Phi_{j}(x),
$$


for some $c_{0}, c_{1}, \ldots, c_{N-1}$, whenever $(x, t) \in V \times(0, T)$. If we extend (3.8) continuously to the domain $V \times[0, T)$, we have,

$$
f(x)=F(x, 0)=\frac{1}{\sqrt{d_{\omega} x}} \sum_{j=0}^{N-1} c_{j} \Phi_{j}(x) .
$$

so that the constants $c_{0}, \ldots, c_{N-1}$ are determined by

$$
c_{j}=\left\langle\sqrt{d_{\omega} y} f(y), \Phi_{j}(y)\right\rangle_{y \in V}=\sum_{y \in V} f(y) \Phi_{j}(y) \sqrt{d_{\omega} y}
$$

for $j=0, \ldots, N-1$. Thus we have

$$
\begin{aligned}
F(x, t) & =\frac{1}{\sqrt{d_{\omega} x}} \sum_{j=0}^{N-1} \sum_{y \in V} f(y) \Phi_{j}(y) \sqrt{d_{\omega} y} e^{-\lambda_{j} t} \Phi_{j}(x) \\
& +\frac{1}{\sqrt{d_{\omega} x}} \sum_{j=0}^{N-1} \sum_{y \in V}\left[\int_{0}^{t} e^{\lambda_{j} \tau} H(y, \tau) d \tau\right] \Phi_{j}(y) \sqrt{d_{\omega} y} e^{-\lambda_{j} t} \Phi_{j}(x) \\
& =\sum_{y \in V} E_{\omega}(x, y, t) f(y)+\sum_{y \in V} \int_{0}^{t} E_{\omega}(x, y, t-\tau) H(y, \tau) d \tau .
\end{aligned}
$$

Hence, if a solution in $\mathcal{C}^{0}(V \times[0, T))$ of equation (3.6) exists, then the solution would be (3.7). Now, it is easy to check the function (3.7) is a solution of the Cauchy problem (3.6).

If the equation in (3.6) is homogeneous, we have the following result.

Corollary 3.4. $\quad$ Let $G(V, E)$ be a graph with a weight $\omega$ and $f: V \rightarrow \mathbf{R}$ be given. Then the unique solution $F(x, t) \in C^{0}(V \times[0, T))$ of the following Cauchy problem

$$
\left\{\begin{array}{l}
\partial_{t} F(x, t)-\Delta_{\omega} F(x, t)=0, \quad x \in V, t \in(0, T) \\
F(x, 0)=f(x), \quad x \in V
\end{array}\right.
$$

is given by

$$
F(x, t)=\left\langle E_{\omega}(x, \cdot, t), f\right\rangle_{V}, \quad x \in V, t \in[0, T) .
$$

Proof. It follows immediately from Theorem 3.3 with $H(x, t) \equiv 0$.

In other words, the continuous solution of the Cauchy problem on the homogeneous $\omega$-diffusion equation can be represented by the inner product of its initial vector and the $\omega$-diffusion kernel. 
Let us now turn to the boundary value problems. For a subgraph $S$ of a host graph $G$ with a weight $\omega$, the Dirichlet eigenvalues of $-\mathcal{L}_{\omega}=D^{1 / 2} \Delta_{\omega} \times$ $D^{-1 / 2}$ are defined to be the eigenvalues

$$
\nu_{1} \leq \nu_{2} \leq \cdots \leq \nu_{n}
$$

of the matrix $-\mathcal{L}_{\omega, S}$ where $\mathcal{L}_{\omega, S}$ is a submatrix of $\mathcal{L}_{\omega}$ with rows and columns restricted to those indexed by vertices in $S$ and $n=|S|$. Let $\phi_{1}, \phi_{2}, \ldots, \phi_{n}$ be the linearly independent functions on $\bar{S}$ such that for each $j=1,2, \ldots, n$,

$$
\mathcal{L}_{\omega, S} \phi_{j}(x)=\left(-\nu_{j}\right) \phi_{j}(x), \quad x \in S \quad \text { and }\left.\quad \phi_{j}\right|_{\partial S}=0 .
$$

In fact, $\phi_{1}, \phi_{2}, \ldots, \phi_{n}$ are the eigenfunctions corresponding to $\nu_{1} \leq \nu_{2} \leq \cdots \leq$ $\nu_{n}$ and can be assumed to be orthonormal in the same sense as above, namely, that for each pair of distinct $i$ and $j$

$$
\sum_{x \in S} \phi_{i}(x) \cdot \phi_{j}(x)=0
$$

while, for all $j$,

$$
\sum_{x \in S}\left|\phi_{j}(x)\right|^{2}=1
$$

As usual, it is known that the first eigenvalue $\nu_{1}>0$.

One can follow now the standard procedure to define Dirichlet $\omega$-diffusion kernel $E_{\omega, S}$ as follows:

$$
E_{\omega, S}(x, y, t)=\sum_{j=1}^{|S|} e^{-\nu_{j} t} \phi_{j}(x) \phi_{j}(y) \frac{\sqrt{d_{\omega} y}}{\sqrt{d_{\omega} x}}, \quad x, y \in S, t \in[0, T) .
$$

We are now ready to solve the Dirichlet boundary value problem (DBVP) of the $\omega$-diffusion equations on graphs. We start with the homogeneous case so that readers can understand the essence of the idea of proof easily.

Theorem 3.4. Let $S$ be a subgraph of a host graph $G$ with a weight $\omega$ with $\partial S \neq \emptyset, \sigma: \partial S \times[0, T) \rightarrow \mathbf{R}$ be a function belong to $\mathcal{C}^{0}(\partial S \times$ $(0, T)) \cap L^{1}(\partial S \times(0, T))$ and $f: S \rightarrow \mathbf{R}$ be given. Then the unique solution $F(x, t) \in \mathcal{C}^{0}(\bar{S} \times(0, T))$ of the following Dirichlet boundary value problem

$$
\left\{\begin{array}{l}
\partial_{t} F(x, t)-\Delta_{\omega} F(x, t)=0, \quad x \in S, t \in(0, T) \\
F(z, t)=\sigma(z, t), \quad z \in \partial S, \quad t \in[0, T) \\
F(x, 0)=f(x), \quad x \in S
\end{array}\right.
$$


is given by

$$
F(x, t)=\left\langle E_{\omega, S}(x, \cdot, t), f\right\rangle_{S}+\int_{0}^{t}\left\langle E_{\omega, S}(x, \cdot, t-\tau), B_{\sigma}(\cdot, \tau)\right\rangle_{S} d \tau
$$

for $x \in S$ and $t \in[0, T)$ with

$$
B_{\sigma}(y, t)=\sum_{z \in \partial S} \frac{\sigma(z, t) \omega(y, z)}{d_{\omega} y}, \quad y \in S, t \in[0, T) .
$$

Proof. Let $n=|S|$ and $D_{S}$ stand for the diagonal matrix whose $x$-th entry is $d_{\omega} x$ for each $x \in S$. Let $F: \bar{S} \times(0, T) \rightarrow \mathbf{R}$ be a solution in $\mathcal{C}_{0}(\bar{S} \times(0, T))$ of the DBVP in (3.12) and consider the expansion

$$
\left.D_{S}^{1 / 2} F\right|_{S}(x, t)=\sum_{j=1}^{n} a_{j}(t) \phi_{j}(x), \quad x \in S, t \in(0, T),
$$

where

$$
a_{j}(t)=\left\langle\left. D_{S}^{1 / 2} F\right|_{S}(\cdot, t), \phi_{j}\right\rangle_{S}=\left\langle D^{1 / 2} F(\cdot, t), \phi_{j}\right\rangle_{\bar{S}},
$$

for $j=1,2, \ldots, n$. Since

$$
\mathcal{L}_{\omega} D^{1 / 2}=D^{1 / 2} \Delta_{\omega}
$$

and

$$
\partial_{t} F(x, t)=\Delta_{\omega} F(x, t),(x, t) \in S \times(0, T),
$$

we have for $t \in(0, T)$,

$$
\begin{aligned}
-\nu_{j} a_{j}(t) & =\left\langle\left. D_{S}^{1 / 2} F\right|_{S}(\cdot, t),-\nu_{j} \phi_{j}\right\rangle_{S} \\
& =\left\langle\left. D_{S}^{1 / 2} F\right|_{S}(\cdot, t), \mathcal{L}_{\omega, S} \phi_{j}\right\rangle_{S} \\
& =\left\langle D^{1 / 2} F(\cdot, t), \mathcal{L}_{\omega} \phi_{j}\right\rangle_{\bar{S}}-\left\langle D^{1 / 2} F(\cdot, t), \mathcal{L}_{\omega} \phi_{j}\right\rangle_{\partial S} \\
& =\left\langle D^{1 / 2} \Delta F(\cdot, t), \phi_{j}\right\rangle_{\bar{S}}-\sum_{y \in S} \sum_{z \in \partial S} \sigma(z, t) \frac{w(y, z)}{\sqrt{d_{\omega} y}} \phi_{j}(y) \\
& =a_{j}^{\prime}(t)-\sum_{y \in S} \sum_{z \in \partial S} \sigma(z, t) \frac{w(y, z)}{\sqrt{d_{\omega} y}} \phi_{j}(y) .
\end{aligned}
$$

Thus there exist constants $c_{1}, c_{2}, \ldots, c_{n}$ such that

$$
a_{j}(t)=c_{j} e^{-\nu_{j} t}+e^{-\nu_{j} t} \sum_{y \in S} \sum_{z \in \partial S}\left[\int_{0}^{t} \sigma(z, \tau) e^{\nu_{j} \tau} d \tau\right] \frac{w(y, z)}{\sqrt{d_{\omega} y}} \phi_{j}(y),
$$


for $t \in(0, T)$ and $j=1,2, \ldots, n$. By extending (3.14) continuously to the domain $S \times[0, T)$, we have

$$
\left\langle\left. D_{S}^{1 / 2} f\right|_{S}, \phi_{j}\right\rangle_{S}=a_{j}(0)=c_{j}
$$

Thus we have

$$
\begin{aligned}
F(x, t) & =\frac{1}{\sqrt{d_{\omega} x}} \sum_{j=1}^{n} a_{j}(t) \phi_{j}(x) \\
& =\sum_{y \in S} E_{\omega, S}(x, y, t) f(y)+\int_{0}^{t} \sum_{y \in S} E_{\omega, S}(x, y, t-\tau) \sum_{z \in \partial S} \sigma(z, \tau) \frac{w(y, z)}{d_{\omega} y} d \tau
\end{aligned}
$$

for $x \in S$ and $t \in[0, T)$, which implies that if there is a solution in $\mathcal{C}^{0}(S \times[0, T))$ of the equation (3.12) then it would be (3.13). A simple calculation shows that the function $F(x, t)$ defined by (3.13) in $S \times[0, T)$ and $F(z, t)=\sigma(z, t)$ in $\partial S \times[0, T)$ gives a solution of the equation (3.12).

The nonhomogeneous case of the DBVP on the $\omega$-diffusion equation can be solved in a similar way as in the above theorem. We state it without proof.

Theorem 3.5. $\quad$ Let $S$ be a subgraph of a host graph $G$ with a weight $\omega$ with $\partial S \neq \emptyset, H: S \times[0, T) \rightarrow \mathbf{R}$ be a function in $\mathcal{C}^{0}(S \times(0, T)) \cap L^{1}(S \times(0, T))$, $\sigma: \partial S \times[0, T) \rightarrow \mathbf{R}$ belong to $\mathcal{C}^{0}(\partial S \times(0, T)) \cap L^{1}(\partial S \times(0, T))$ and $f: S \rightarrow \mathbf{R}$ be given. Then the unique solution $F(x, t) \in \mathcal{C}^{0}(S \times(0, T))$ of the following Dirichlet boundary value problem

$$
\left\{\begin{array}{l}
\partial_{t} F(x, t)-\Delta_{\omega} F(x, t)=H(x, t), \quad x \in S, t \in(0, T) \\
F(z, t)=\sigma(z, t), \quad z \in \partial S, t \in[0, T) \\
F(x, 0)=f(x), \quad x \in S
\end{array}\right.
$$

is given by

$$
F(x, t)=\left\langle E_{\omega, S}(x, \cdot, t), f\right\rangle_{S}+\int_{0}^{t}\left\langle E_{\omega, S}(x, \cdot, t-\tau), H(\cdot, \tau)+B_{\sigma}(\cdot, \tau)\right\rangle_{S} d \tau
$$

for $x \in S$ and $t \in[0, T)$ with

$$
B_{\sigma}(y, t)=\sum_{z \in \partial S} \frac{\sigma(z, t) \omega(y, z)}{d_{\omega} y}, \quad y \in S, t \in[0, T) .
$$

As an application of Corollary 3.3, we give the following Huygens property of the homogeneous $\omega$-diffusion equation. 
Theorem 3.6. Let $G=G(V, E)$ be a graph with a weight $\omega$ and $F$ : $V \times(0, T) \rightarrow \mathbf{R}$ be a function. If

$$
\partial_{t} F(x, t)-\Delta_{\omega} F(x, t)=0, \quad x \in V, t \in(0, T),
$$

then for every $t$ and $\delta \in(0, T)$ with $t+\delta<T$, we have

$$
F(x, t+\delta)=\left\langle E_{\omega}(x, \cdot, \delta), F(\cdot, t)\right\rangle_{V} .
$$

Proof. Let $N=|V|$. By Corollary 3.3, we can expand the function F as

$$
F(x, t)=\frac{1}{\sqrt{d_{\omega} x}} \sum_{j=0}^{N-1} c_{j} e^{-\lambda_{j} t} \Phi_{j}(x),
$$

for some constants $c_{0}, c_{1}, \ldots, c_{N-1}$. Hence

$$
\begin{aligned}
F(x, t+\delta) & =\frac{1}{\sqrt{d_{\omega} x}} \sum_{j=0}^{N-1} c_{j} e^{-\lambda_{j}(t+\delta)} \Phi_{j}(x) \\
& =\frac{1}{\sqrt{d_{\omega} x}} \sum_{j=0}^{N-1} e^{-\lambda_{j} \delta} c_{j} e^{-\lambda_{j} t} \Phi_{j}(x) \\
& =\frac{1}{\sqrt{d_{\omega} x}} \sum_{j=0}^{N-1} e^{-\lambda_{j} \delta} \Phi_{j}(x)\left\langle\sqrt{d_{\omega} y} \Phi_{j}(y), \frac{1}{\sqrt{d_{\omega} y}} \sum_{k=1}^{N-1} c_{k} e^{-\lambda_{k} t} \Phi_{k}(y)\right\rangle_{y \in V} \\
& =\frac{1}{\sqrt{d_{\omega} x}} \sum_{j=0}^{N-1} e^{-\lambda_{j} \delta} \Phi_{j}(x)\left\langle\sqrt{d_{\omega} y} \Phi_{j}(y), F(y, t)\right\rangle_{y \in V} \\
& =\left\langle E_{\omega}(x, \cdot, \delta), F(\cdot, t)\right\rangle_{V} .
\end{aligned}
$$

So far, we have discussed the uniqueness of the solutions of the DBVP of the $\omega$-diffusion equations by two methods, one is by using the minimum and maximum principle and the other is by constructing the solution directly. Here, we provide an alternative argument based upon energy method, the idea of which can be found in the book [E] written by L. C. Evans, which deals with the theory of the (classical) partial differential equations.

Theorem 3.7. $\quad$ Let $S$ be a subgraph of a host graph with a weight $\omega$ such that $\partial S \neq \emptyset$. If a function $F \in \mathcal{C}^{0}(\bar{S} \times[0, T))$ is a solution of the following $D B V P$

$$
\left\{\begin{array}{l}
\partial_{t} F(x, t)-\Delta_{\omega} F(x, t)=0, \quad x \in S, t \in(0, T) \\
F(z, t)=0, \quad z \in \partial S, \quad t \in[0, T) \\
F(x, 0)=0, \quad x \in S
\end{array}\right.
$$


then $F(x, t) \equiv 0$ on $\bar{S} \times[0, T)$.

Proof. Let

$$
e(t):=\int_{\bar{S}} F^{2}(x, t) d_{\omega} x, \quad t \in[0, T) .
$$

Then we have

$$
\begin{aligned}
\frac{d}{d t} e(t) & =2 \int_{\bar{S}} F(x, t) \cdot \partial_{t} F(x, t) d_{\omega} x \\
& =2 \int_{\bar{S}} F(x, t) \cdot \Delta_{\omega} F(x, t) d_{\omega} x \\
& =-\int_{\bar{S}}\left|\nabla_{\omega} F(x, t)\right|^{2} d_{\omega} x \leq 0, \quad t \in(0, T) .
\end{aligned}
$$

Therefore

$$
0 \leq e(t) \leq e(0)=0, \quad t \in[0, T),
$$

which implies $F(x, t) \equiv 0$ on $\bar{S} \times[0, T)$.

Remark. In the book [E], it is discussed to prove the uniqueness of the solutions of the DBVP on (classical) heat equation via energy method. The basic idea is to define an energy at time $t \geq 0$ of the solutions of the heat equation by means of integration and then prove that the energy is constant if the boundary value of the solution is constantly zero, by using Green's formula. Since the discrete version of integration and Green's formula was recently introduced in [CB], authors thought it would be possible to apply the method in [E] to the discrete case.

\section{$\S 4$. $\omega$-Elastic Equations on Weighted Graphs}

In this section we investigate the $\omega$-elastic equation on graphs, subject to the appropriate initial and boundary conditions.

For a graph $G(V, E)$ with a weight $\omega$ we say that a function $F: V \times(0, T) \rightarrow$ $\mathbf{R}$ satisfies $\omega$-elastic equation on $V \times(0, T)$ if it satisfies

$$
\partial_{t}^{2} F(x, t)-\Delta_{\omega} F(x, t)=H(x, t), \quad x \in S, t \in(0, T),
$$

for a given function $H(x, t)$ in $V \times(0, T)$. If $H(x, t) \equiv 0$ then we say that the equation is homogeneous.

We give a physical model on $\omega$-elastic equation. Let us consider a set of atoms lying on the vertices of a weighted graph. A function $\mathbf{F}: V \times(0, T) \rightarrow \mathbf{R}^{3}$ 
represents the displacement of the atom on the vertex $x$ at time $t \in(0, T)$. Each atom acts on its neighboring atoms by elastic forces proportional to elasticity and distance between each atoms, which can be written as $\sum_{y \in V}[\mathbf{F}(y, t)-$ $\mathbf{F}(x, t)] \omega(x, y)$, where $\omega(x, y)$ represents the elasticity between the atoms lying in vertices $x$ and $y$. Since this elastic forces cause acceleration, it is easy to see that the function $\mathbf{F}$ satisfies the equation

$$
\partial_{t}^{2} \mathbf{F}(x, t)-\Delta_{\omega} \mathbf{F}(x, t)=0, \quad x \in V, t \in(0, T),
$$

each of whose component is the homogeneous $\omega$-elastic equation.

We first consider the solution of the $\omega$-elastic equation without boundary condition.

Theorem 4.1. Let $G(V, E)$ be a graph with a weight $\omega$ and $H(x, t) \in$ $\mathcal{C}^{0}(V \times(0, T)) \cap L^{1}(V \times(0, T))$. Then every solution $F(x, t)$ of the equation

$$
\partial_{t}^{2} F(x, t)-\Delta_{\omega} F(x, t)=H(x, t), \quad x \in V, t \in(0, T)
$$

is represented as follows: there exist $c_{0}, c_{1}, \ldots, c_{N-1}, d_{0}, d_{1}, \ldots, d_{N-1}$ such that

$$
F(x, t)=\frac{1}{\sqrt{d_{\omega} x}} \sum_{j=0}^{N-1} a_{j}(t) \Phi_{j}(x), \quad x \in V, t \in(0, T),
$$

where

$$
\left.a_{0}(t)=c_{0}+d_{0} t+\int_{0}^{t}(t-\tau)\left\langle D^{1 / 2} H(\cdot, t), \Phi_{0}\right)\right\rangle_{V} d \tau, \quad t \in(0, T)
$$

and

$$
\begin{aligned}
a_{j}(t) & =c_{j} \cos \sqrt{\lambda_{j}} t+d_{j} \sin \sqrt{\lambda_{j}} t \\
& \left.+\frac{1}{\sqrt{\lambda_{j}}} \int_{0}^{t} \sin \left(\sqrt{\lambda_{j}}(t-\tau)\right)\left\langle D^{1 / 2} H(\cdot, t), \Phi_{j}\right)\right\rangle_{V} d \tau, \quad t \in(0, T),
\end{aligned}
$$

for $j=1,2, \ldots, N-1$, where $N=|V|$.

Proof. Consider the expansions

$$
\sqrt{d_{\omega} x} F(x, t)=\sum_{j=0}^{N-1} a_{j}(t) \Phi_{j}(x), \quad j=1,2, \ldots, N-1
$$


and

$$
\sqrt{d_{\omega} x} H(x, t)=\sum_{j=0}^{N-1} b_{j}(t) \Phi_{j}(x), \quad j=1,2, \ldots, N-1,
$$

where $a_{j}(t)=\left\langle D^{1 / 2} F(\cdot, t), \Phi_{j}\right\rangle_{V}$ and $b_{j}(t)=\left\langle D^{1 / 2} H(\cdot, t), \Phi_{j}\right\rangle_{V}$. Then since $\mathcal{L}_{\omega} D^{1 / 2}=D^{1 / 2} \Delta_{\omega}$ and

$$
\begin{aligned}
-\lambda_{j} a_{j}(t) & =\left\langle D^{1 / 2} F(\cdot, t), \mathcal{L}_{\omega} \Phi_{j}\right\rangle_{V} \\
& =\left\langle\mathcal{L}_{\omega} D^{1 / 2} F(\cdot, t), \Phi_{j}\right\rangle_{V} \\
& =\left\langle D^{1 / 2}\left[\partial_{t}^{2} F(\cdot, t)-H(\cdot, t)\right], \Phi_{j}\right\rangle_{V} \\
& =a_{j}^{\prime \prime}(t)-\left\langle D^{1 / 2} H(\cdot, t), \Phi_{j}\right\rangle_{V}, \\
& =a_{j}^{\prime \prime}(t)-b_{j}(t), \quad t \in(0, T)
\end{aligned}
$$

we have

$$
a_{j}^{\prime \prime}(t)+\lambda_{j} a_{j}(t)=b_{j}(t), \quad t \in(0, T)
$$

for all $j=0,1, \ldots, N-1$. Solving the differential equations (4.3), we have the homogeneous solutions

$$
a_{h, j}(t)=\left\{\begin{array}{l}
c_{0}+d_{0} t, \quad j=0 \\
c_{j} \cos \sqrt{\lambda_{j}} t+d_{j} \sin \sqrt{\lambda_{j}} t, j=1,2, \ldots, N-1,
\end{array}\right.
$$

where $c_{0}, c_{1}, \ldots, c_{N-1}, d_{0}, d_{1}, \ldots, d_{N-1}$ and the particular solutions

$$
a_{p, j}(t)= \begin{cases}\int_{0}^{t}(t-\tau) b_{0}(\tau) d \tau, & j=0 \\ \frac{1}{\sqrt{\lambda_{j}}} \int_{0}^{t} \sin \left(\sqrt{\lambda_{j}}(t-\tau)\right) b_{j}(\tau) d \tau, & j=1,2, \ldots, N-1 .\end{cases}
$$

Thus we have

$$
a_{0}(t)=c_{0}+d_{0} t+\int_{0}^{t}(t-\tau) b_{0}(\tau) d \tau, \quad t \in(0, T)
$$

and

$$
\begin{aligned}
a_{j}(t) & =c_{j} \cos \sqrt{\lambda_{j}} t+d_{j} \sin \sqrt{\lambda_{j}} t \\
& +\frac{1}{\sqrt{\lambda_{j}}} \int_{0}^{t} \sin \left(\sqrt{\lambda_{j}}(t-\tau)\right) b_{j}(\tau) d \tau, \quad t \in(0, T),
\end{aligned}
$$

for $j=1,2, \ldots, N-1$. Therefore if the equation (4.1) has a solution, then it must be given as (4.2). Now, it is a simple manipulation to prove the function (4.2) satisfies the equation (4.1).

Letting $H(x, t) \equiv 0$ in Theorem 4.1 we obtain the following solutions of the homogeneous $\omega$-elastic equation. 
Corollary 4.1. Let $G=G(V, E)$ be a graph with a weight $\omega$. Every solution $F(x, t)$ of the equation

$$
\partial_{t}^{2} F(x, t)-\Delta_{\omega} F(x, t)=0, \quad x \in V, t \in(0, T),
$$

can be represented as follows: there exist $c_{0}, c_{1}, \ldots, c_{N-1}, d_{0}, d_{1}, \ldots, d_{N-1}$ such that

$$
F(x, t)=\frac{1}{\sqrt{d_{\omega} x}}\left[\left(c_{0}+d_{0} t\right) \Phi_{0}(x)+\sum_{j=1}^{N-1} c_{j} \cos \sqrt{\lambda_{j}} t+d_{j} \sin \sqrt{\lambda_{j}} t\right],
$$

for $x \in V$ and $t \in(0, T)$, where $N=|V|$.

For a graph $G(V, E)$ with a weight $\omega$, the function $W_{\omega}: V \times V \times[0, T) \rightarrow \mathbf{R}$ defined by

$$
\begin{aligned}
& W_{\omega}(x, y, t) \\
& \quad=\left[t \Phi_{0}(x) \Phi_{0}(y)+\sum_{j=1}^{|V|-1} \frac{1}{\sqrt{\lambda_{j}}} \sin \left(\sqrt{\lambda_{j}} t\right) \Phi_{j}(x) \Phi_{j}(y)\right] \sqrt{\frac{d_{\omega} y}{d_{\omega} x}},
\end{aligned}
$$

is called the $\omega$-elastic kernel.

Remark. By virtue of the fact that $\lim _{x \rightarrow 0} \sin (t x) / x=t$, we sometimes denote (4.4) as

$$
W_{\omega}(x, y, t)=\sum_{j=0}^{|V|-1} \frac{1}{\sqrt{\lambda_{j}}} \sin \sqrt{\lambda_{j}} t \Phi_{j}(x) \Phi_{j}(y) \sqrt{\frac{d_{\omega} y}{d_{\omega} x}},
$$

for simplicity.

By using the result in Theorem 4.1, we solve the following Cauchy problem of the $\omega$-elastic equations and represent the solutions by the $\omega$-elastic kernel.

Theorem 4.2. Let $G(V, E)$ be a graph with a weight $\omega, H: V \times[0, T) \rightarrow$ $\mathbf{R}$ be a function belong to $\mathcal{C}^{0}(V \times(0, T)) \bigcap L^{1}(V \times(0, T))$ and $f, g: V \rightarrow \mathbf{R}$ be given. Then the unique solution $F(x, t) \in \mathcal{C}^{1}(V \times[0, T))$ of the following Cauchy problem

$$
\left\{\begin{array}{l}
\partial_{t}^{2} F(x, t)-\Delta_{\omega} F(x, t)=H(x), \quad x \in V, t \in(0, T) \\
F(x, 0)=f(x), \quad x \in V \\
\partial_{t} F(x, 0)=g(x), \quad x \in V
\end{array}\right.
$$


is given by

$$
\begin{aligned}
F(x, t)= & \left\langle W_{\omega}(x, \cdot, t), g\right\rangle_{V}+\left\langle\partial_{t} W_{\omega}(x, \cdot, t), f\right\rangle_{V} \\
& +\int_{0}^{t}\left\langle W_{\omega}(x, \cdot, t-\tau), H(\cdot, \tau)\right\rangle_{V} d \tau
\end{aligned}
$$

for $x, y \in V$ and $t \in[0, T)$, with $N=|V|$.

Proof. Let $F(x, t)$ be a solution in $\mathcal{C}^{1}(V \times[0, T))$ of the equation (4.5). By Theorem 4.1, there exist real constants $c_{0}, \ldots, c_{N-1}, d_{0}, \ldots, d_{N-1}$ such that

$$
F(x, t)=\frac{1}{\sqrt{d_{\omega} x}} \sum_{j=0}^{N-1} a_{j}(t) \Phi_{j}(x), \quad(x, t) \in V \times(0, T)
$$

where

$$
\left.a_{0}(t)=c_{0}+d_{0} t+\int_{0}^{t}(t-\tau)\left\langle D^{1 / 2} H(\cdot, t), \Phi_{0}\right)\right\rangle_{V} d \tau, \quad t \in(0, T)
$$

and

$$
\begin{aligned}
a_{j}(t)= & c_{j} \cos \sqrt{\lambda_{j}} t+d_{j} \sin \sqrt{\lambda_{j}} t \\
& \left.+\frac{1}{\sqrt{\lambda_{j}}} \int_{0}^{t} \sin \left(\sqrt{\lambda_{j}}(t-\tau)\right)\left\langle D^{1 / 2} H(\cdot, t), \Phi_{j}\right)\right\rangle_{V} d \tau, \quad t \in(0, T),
\end{aligned}
$$

for $j=1,2, \ldots, N-1$. Then it follows from the assumption $F(x, t) \in C^{1}(V \times$ $[0, T))$ that

$$
f(x)=F(x, 0)=\lim _{t \rightarrow 0} F(x, t)=\frac{1}{\sqrt{d_{\omega} x}} \sum_{j=0}^{N-1} c_{j} \Phi_{j}(x) .
$$

Hence the constants $c_{0}, c_{1}, \ldots, c_{n-1}$ are determined by

$$
c_{j}=\left\langle\sqrt{d_{\omega} y} f(y), \Phi_{j}(y)\right\rangle_{y \in V}=\sum_{y \in V} f(y) \Phi_{j}(y) \sqrt{d_{\omega} y}
$$

Thus we have

$$
\begin{aligned}
c_{0} \frac{\Phi_{0}(x)}{\sqrt{d_{\omega} x}}+\sum_{j=1}^{N-1} c_{j} \cos \sqrt{\lambda_{j}} t \frac{\Phi_{j}(x)}{\sqrt{d_{\omega} x}} & =\left\langle f(y), \sum_{j=0}^{N-1} \cos \sqrt{\lambda_{j}} t \Phi_{j}(x) \Phi_{j}(y) \frac{\sqrt{d_{\omega} y}}{\sqrt{d_{\omega} x}}\right\rangle_{y \in V} \\
& =\left\langle\partial_{t} W_{\omega}(x, \cdot, t), f\right\rangle_{V}
\end{aligned}
$$


for $x \in V$ and $t \in[0, T)$. On the other hand, it again follows from the assumption $F(x, t) \in \mathcal{C}^{1}(V \times[0, T))$ that

$$
g(x)=\partial_{t} F(x, 0)=\lim _{t \rightarrow 0} \partial_{t} F(x, t)=\frac{1}{\sqrt{d_{\omega} x}} \sum_{j=0}^{N-1} \sqrt{\lambda_{j}} d_{j} \Phi_{j}(x),
$$

and so $d_{0}, d_{1}, \ldots, d_{n-1}$ are determined by

$$
d_{j}=\left\{\begin{array}{l}
\left\langle\sqrt{d_{\omega} y} g(y), \Phi_{0}(y)\right\rangle_{y \in V}, \quad j=0 \\
\frac{1}{\sqrt{\lambda_{j}}}\left\langle\sqrt{d_{\omega} y} g(y), \Phi_{j}(y)\right\rangle_{y \in V}, j=1,2, \ldots, N-1 .
\end{array}\right.
$$

Therefore we have

$$
\begin{aligned}
d_{0} t \frac{\Phi_{0}(x)}{\sqrt{d_{\omega} x}} & +\sum_{j=1}^{N-1} d_{j} \sin \sqrt{\lambda_{j}} t \frac{\Phi_{j}(x)}{\sqrt{d_{\omega} x}} \\
& =\left\langle g(y),\left[t \Phi_{0}(x) \Phi_{0}(y)+\sum_{j=1}^{N-1} \frac{1}{\sqrt{\lambda_{j}}} \sin \sqrt{\lambda_{j}} t \Phi_{j}(x) \Phi_{j}(y)\right] \sqrt{\frac{d_{\omega} y}{d_{\omega} x}}\right\rangle_{y \in V} \\
& =\left\langle W_{\omega}(x, \cdot, t), g\right\rangle_{V},
\end{aligned}
$$

for $x \in V$ and $t \in[0, T)$. Finally, we have

$$
\begin{aligned}
\frac{1}{\sqrt{d_{\omega} x}} & {\left[\int_{0}^{t}(t-\tau)\left\langle D^{1 / 2} H(\cdot, t), \Phi_{0}\right)\right\rangle_{V} d \tau } \\
& \left.\left.+\int_{0}^{t} \sin \left(\sqrt{\lambda_{j}}(t-\tau)\right)\left\langle D^{1 / 2} H(\cdot, t), \Phi_{j}\right)\right\rangle_{V} d \tau\right] \\
& =\int_{0}^{t}\left\langle W_{\omega}(x, \cdot, t-\tau), H(\cdot, \tau)\right\rangle_{V} d \tau, \quad x \in V, t \in[0, T)
\end{aligned}
$$

which implies that if equation (4.5) has a solution in $\mathcal{C}^{1}(V \times[0, T))$, then it would be (4.6). Conversely, it is easy to see that the function in (4.6) is a solution of the equation (4.5).

By using the previous result, we solve the following Cauchy problem of homogeneous $\omega$-elastic equation.

Corollary 4.2. $\quad$ Let $G(V, E)$ be a graph with a weight $\omega$ and let functions $f$ and $g: V \rightarrow \mathbf{R}$ be given. Then the unique solution $F(x, t) \in \mathcal{C}^{1}(V \times[0, T))$ of the following Cauchy problem

$$
\left\{\begin{array}{l}
\partial_{t}^{2} F(x, t)-\Delta_{\omega} F(x, t)=0, \quad x \in V, t \in(0, T) \\
F(x, 0)=f(x), \quad x \in V \\
\partial_{t} F(x, 0)=g(x)
\end{array}\right.
$$


is given by

$$
F(x, t)=\left\langle W_{\omega}(x, \cdot, t), g\right\rangle_{V}+\left\langle\partial_{t} W_{\omega}(x, \cdot, t), f\right\rangle_{V}
$$

Proof. This is the case of $H(x, t) \equiv 0$, in the previous theorem.

We now turn our attention to the boundary value problem. We again employ the Dirichlet eigenvalues $\nu_{1}, \nu_{2}, \ldots, \nu_{n}$ and eigenfunctions $\phi_{1}, \phi_{2}, \ldots, \phi_{n}$ as in the previous section. Similar to the case of the $\omega$-diffusion kernel in the previous section, we define the Dirichlet $\omega$-elastic kernel, $W_{\omega, S}(x, y, t)$, as follows:

$$
W_{\omega, S}(x, y, t)=\left[t \phi_{0}(x) \phi_{0}(y)+\sum_{j=1}^{|S|} \frac{1}{\sqrt{\nu_{j}}} \sin \left(\sqrt{\nu_{j}} t\right) \phi_{j}(x) \phi_{j}(y)\right] \sqrt{\frac{d_{\omega} y}{d_{\omega} x}},
$$

for $x, y \in S$ and $t \in[0, T)$.

Theorem 4.3. Let $S$ be a subgraph of a host graph $G$ with a weight $\omega$ with $\partial S \neq \emptyset, \sigma: \partial S \times[0, T) \rightarrow \mathbf{R}$ be a function belong to $\mathcal{C}^{0}(\partial S \times$ $(0, T)) \bigcap L^{1}(\partial S \times(0, T))$ and $f, g: S \rightarrow \mathbf{R}$ be given. Then the unique solution $F(x, t) \in \mathcal{C}^{1}(S \times(0, T))$ of the following Dirichlet boundary value problem

$$
\left\{\begin{array}{l}
\partial_{t}^{2} F(x, t)-\Delta_{\omega} F(x, t)=0, \quad x \in S, t \in(0, T) \\
F(z, t)=\sigma(z, t), \quad z \in \partial S, \quad t \in[0, T) \\
F(x, 0)=f(x), \quad x \in S \\
\partial_{t} F(x, 0)=g(x), \quad x \in S
\end{array}\right.
$$

is given by

$$
\begin{aligned}
F(x, t)=\left\langle W_{\omega, S}(x, \cdot, t), g\right\rangle_{S} & +\left\langle\partial_{t} W_{\omega, S}(x, \cdot, t), f\right\rangle_{S} \\
& +\int_{0}^{t}\left\langle W_{\omega, S}(x, \cdot, t-\tau), B_{\sigma}(\cdot, \tau)\right\rangle_{S} d \tau
\end{aligned}
$$

for $x \in S$ and $t \in[0, T)$ with

$$
B_{\sigma}(y, t)=\sum_{z \in \partial S} \frac{\sigma(z, t) \omega(y, z)}{d_{\omega} y}, \quad y \in S, t \in[0, T) .
$$

Proof. Let $n=|S|$ and $F: \bar{S} \times[0, T) \rightarrow \mathbf{R}$ be a solution in $\mathcal{C}^{1}(S \times[0, T))$ of the DBVP (4.8). Consider the expansion

$$
\left.D_{S}^{1 / 2} F\right|_{S}(x, t)=\sum_{j=1}^{n} a_{j}(t) \phi_{j}(x), \quad x \in S, t \in(0, T),
$$


where

$$
a_{j}(t)=\left\langle\left. D_{S}^{1 / 2} F\right|_{S}(\cdot, t), \phi_{j}\right\rangle_{S}=\left\langle D^{1 / 2} F(\cdot, t), \phi_{j}\right\rangle_{\bar{S}},
$$

for $t \in(0, T)$ and $j=1,2, \ldots, n$. Then since

$$
\mathcal{L}_{\omega} D^{1 / 2}=D^{1 / 2} \Delta_{\omega}
$$

and

$$
\partial^{2} F(x, t)=\Delta_{\omega} F(x, t), \quad(x, t) \in S \times(0, T),
$$

we have

$$
\begin{aligned}
-\nu_{j} a_{j}(t) & =\left\langle\left. D_{S}^{1 / 2} F\right|_{S}(\cdot, t),-\nu_{j} \phi_{j}\right\rangle_{S} \\
& =\left\langle\left. D_{S}^{1 / 2} F\right|_{S}(\cdot, t), \mathcal{L}_{\omega, S} \phi_{j}\right\rangle_{S} \\
& =\left\langle D^{1 / 2} F(\cdot, t), \mathcal{L}_{\omega} \phi_{j}\right\rangle_{\bar{S}}-\left\langle D^{1 / 2} F(\cdot, t), \mathcal{L}_{\omega} \phi_{j}\right\rangle_{\partial S} \\
& =\left\langle D^{1 / 2} \Delta F(\cdot, t), \phi_{j}\right\rangle_{\bar{S}}-\sum_{y \in S} \sum_{z \in \partial S} \sigma(z, t) \frac{w(y, z)}{\sqrt{d_{\omega} y}} \phi_{j}(y) \\
& =a_{j}^{\prime \prime}(t)-\sum_{y \in S} \sum_{z \in \partial S} \sigma(z, t) \frac{w(y, z)}{\sqrt{d_{\omega} y}} \phi_{j}(y), t \in(0, T) .
\end{aligned}
$$

Letting

$$
b_{j}(t):=\sum_{y \in S} \sum_{z \in \partial S} \sigma(z, t) \frac{w(y, z)}{\sqrt{d_{\omega} y}}
$$

and solving the above differential equation, we have the homogeneous solutions

$$
a_{h, j}(t)=c_{j} \cos \sqrt{\nu_{j}} t+d_{j} \sin \sqrt{\nu_{j}} t, \quad t \in(0, T)
$$

for some constants $c_{1}, \ldots, c_{n}, d_{1}, \ldots, d_{n}$ and particular solutions

$$
\begin{aligned}
a_{p, j}(t)=-\frac{1}{\sqrt{\nu}_{j}} & \cos \sqrt{\nu}_{j} t \int_{0}^{t} b_{j}(\tau) \sin \sqrt{\nu}_{j} \tau d \tau \\
& +\frac{1}{\sqrt{\nu}_{j}} \sin \sqrt{\nu}_{j} t \int_{0}^{t} b_{j}(\tau) \cos \sqrt{\nu}_{j} \tau d \tau,
\end{aligned}
$$

for $t \in(0, T)$ and $j=1,2, \ldots, n$. Since $F(x, t)$ is in $\mathcal{C}^{1}(S \times[0, T))$, we have

$$
\left\langle\left. D_{S}^{1 / 2} f\right|_{S}, \phi_{j}\right\rangle_{S}=a_{j}(0)=\lim _{t \rightarrow 0} a_{j}(t)=c_{j}
$$

and

$$
\left\langle\left. D_{S}^{1 / 2} g\right|_{S}, \phi_{j}\right\rangle_{S}=a_{j}^{\prime}(0)=\lim _{t \rightarrow 0} a_{j}^{\prime}(t)=d_{j}
$$


Thus we have

$$
\begin{aligned}
F(x, t)= & \frac{1}{\sqrt{d_{\omega} x}} \sum_{j=1}^{n}\left[a_{h, j}(t)+a_{p, j}(t)\right] \phi_{j}(x) \\
= & \left\langle g(y), \sum_{j=1}^{n} \frac{1}{\sqrt{\nu}_{j}} \sin \left(\sqrt{\nu}_{j} t\right) \phi_{j}(x) \phi_{j}(y) \frac{\sqrt{d_{\omega} y}}{\sqrt{d_{\omega} x}}\right\rangle_{y \in S} \\
& +\left\langle f(y), \sum_{j=1}^{n} \cos \left(\sqrt{\nu_{j}} t\right) \phi_{j}(x) \phi_{j}(y) \frac{\sqrt{d_{\omega} y}}{\sqrt{d_{\omega} x}}\right\rangle_{y \in S} \\
& +\int_{0}^{t}\left\langle B_{\sigma}(y, t), \sum_{j=1}^{n} \frac{1}{\sqrt{\nu}_{j}} \sin (t-\tau) \phi_{j}(y) \phi_{j}(x) \frac{\sqrt{d_{\omega} y}}{\sqrt{d_{\omega} x}}\right\rangle_{y \in S} d \tau,
\end{aligned}
$$

which indicates that if the equation (4.8) has a solution in $\mathcal{C}^{1}(S \times[0, T))$ then it would be (4.9). Moreover, a simple calculation shows that the function $F(x, t)$ satisfies the form (4.9) in $S \times[0, T)$ and $F(z, t)=\sigma(z, t)$ in $(z, t) \in$ $\partial S \times[0, T)$.

The DBVP of the nonharmonic $\omega$-elastic equation is given as follows.

Theorem 4.4. Let $S$ be a subgraph of a host graph $G$ with a weight $\omega$ with $\partial S \neq \emptyset, H: S \times[0, T) \rightarrow \mathbf{R}$ be a function in $\mathcal{C}^{0}(S \times(0, T)) \cap L^{1}(S \times(0, T))$, $\sigma: \partial S \times[0, T) \rightarrow \mathbf{R}$ belong to $\mathcal{C}^{0}(\partial S \times(0, T)) \cap L^{1}(\partial S \times(0, T))$ and $f, g: S \rightarrow \mathbf{R}$ be given. Then the unique solution $F(x, t) \in \mathcal{C}^{1}(S \times(0, T))$ of the following Dirichlet boundary value problem

$$
\left\{\begin{array}{l}
\partial_{t}^{2} F(x, t)-\Delta_{\omega} F(x, t)=0, \quad x \in S, t \in(0, T) \\
F(z, t)=\sigma(z, t), \quad z \in \partial S, \quad t \in[0, T) \\
F(x, 0)=f(x), \quad x \in S \\
\partial_{t} F(x, 0)=g(x), \quad x \in S
\end{array}\right.
$$

is represented by

$$
\begin{aligned}
F(x, t)=\left\langle W_{\omega, S}(x, \cdot, t), g\right\rangle_{S} & +\left\langle\partial_{t} W_{\omega, S}(x, \cdot, t), f\right\rangle_{S} \\
& +\int_{0}^{t}\left\langle W_{\omega, S}(x, \cdot, t-\tau), H(\cdot, \tau)+B_{\sigma}(\cdot, \tau)\right\rangle_{S} d \tau,
\end{aligned}
$$

for $x \in S$ and $t \in[0, T)$, where

$$
B_{\sigma}(y, t)=\sum_{z \in \partial S} \frac{\sigma(z, t) \omega(y, z)}{d_{\omega} y}, \quad y \in S, t \in[0, T) .
$$


Proof. Following the proof of Theorem 4.3 with some manipulations gives the result.

Next result gives the Huygens property on homogeneous $\omega$-elastic equation.

Theorem 4.5. Let $G=G(V, E)$ be a graph with a weight $\omega$ and $F$ : $V \times(0, T) \rightarrow \mathbf{R}$ be a function. If

$$
\partial_{t}^{2} F(x, t)-\Delta_{\omega} F(x, t)=0, \quad x \in V \quad t \in(0, T),
$$

then for every $t>0$ and $\delta>0$,

$$
F(x, t+\delta)=\left\langle\partial_{t} W_{\omega}(x, \cdot, \delta), F(\cdot, t)\right\rangle_{V}+\left\langle W_{\omega}(x, \cdot, \delta), \partial_{t} F(\cdot, t)\right\rangle_{V}, \quad x \in V
$$

Proof. Let $N=|V|$. By Corollary 4.1, we can expand the function F as

$$
F(x, t+\delta)=\frac{1}{\sqrt{d_{\omega} x}} \sum_{j=0}^{N-1} a_{j}(t+\delta) \Phi_{j}(x)
$$

for all $x \in V$ and $t, \delta \in(0, \infty)$, where

$$
a_{j}(t+\delta)= \begin{cases}c_{0}+d_{0}(t+\delta), & j=0, \\ c_{j} \cos \left(\sqrt{\lambda_{j}}(t+\delta)\right)+d_{j} \sin \left(\sqrt{\lambda_{j}}(t+\delta)\right), & j=1, \ldots, N-1,\end{cases}
$$

for some constants $c_{0}, \ldots, c_{N-1}, d_{0}, \ldots, d_{N-1}$. Since

$$
\begin{aligned}
a_{j}(t+\delta)= & \left(c_{j} \cos \sqrt{\lambda_{j}} t+d_{j} \sin \sqrt{\lambda_{j}} t\right) \cos \sqrt{\lambda_{j}} \delta \\
& +\left(-c_{j} \sin \sqrt{\lambda_{j}} t+d_{j} \cos \sqrt{\lambda_{j}} t\right) \sin \sqrt{\lambda_{j}} \delta \\
= & a_{j}(t) \cos \sqrt{\lambda_{j}} \delta+\frac{1}{\sqrt{\lambda}_{j}} a_{j}^{\prime}(t) \sin \sqrt{\lambda_{j}} \delta, \quad t, \delta \in(0, T)
\end{aligned}
$$

for $j=1, \ldots, N-1$ and

$$
\begin{aligned}
a_{0}(t+\delta) & =\left(c_{0}+d_{0} t\right) \cos \sqrt{\lambda_{0}} \delta+\frac{d_{0}}{\sqrt{\lambda_{0}}} \sin \sqrt{\lambda_{0}} \delta \\
& =a_{0}(t) \cos \sqrt{\lambda_{0}} \delta+\frac{1}{\sqrt{\lambda}_{0}} a_{0}^{\prime}(t) \sin \sqrt{\lambda_{0}} \delta, \quad t, \delta \in(0, T),
\end{aligned}
$$


we obtain

$$
\begin{aligned}
F(x, t+\delta)= & \frac{1}{\sqrt{d_{\omega} x}}\left[\sum_{j=0}^{N-1} a_{j}(t) \Phi_{j}(x)+\sum_{j=0}^{N-1} a_{j}^{\prime}(\delta) \Phi_{j}(x)\right] \\
= & \left\langle F(y, t), \sum_{j=0}^{N-1} \cos \left(\sqrt{\lambda_{j}} \delta\right) \Phi_{j}(x) \Phi_{j}(y) \frac{\sqrt{d_{\omega} y}}{\sqrt{d_{\omega} x}}\right\rangle_{y \in V} \\
& +\left\langle\partial_{t} F(y, t), \sum_{j=0}^{N-1} \frac{1}{\sqrt{\lambda_{j}}} \cos \left(\sqrt{\lambda_{j} \delta}\right) \Phi_{j}(x) \Phi_{j}(y) \frac{\sqrt{d_{\omega} y}}{\sqrt{d_{\omega} x}}\right\rangle_{y \in V} \\
= & \left\langle F(\cdot, t), \partial_{t} W_{\omega}(x, \cdot, \delta)\right\rangle_{V}+\left\langle\partial_{t} F(\cdot, t), W_{\omega}(x, \cdot, \delta)\right\rangle_{V},
\end{aligned}
$$

for $x \in V$ and $t, \delta \in(0, T)$.

In the remaining of this section, we see that the $\omega$-elastic equation is nicely behaved with respect to an integral energy norm.

Let $S$ be a subgraph of a host graph. For a function $F \in \mathcal{C}^{1}(\bar{S} \times[0, T))$, we define the "energy" of the function $F$ as following:

$$
\begin{aligned}
\mathbf{e}(t): & =\int_{\bar{S}}\left[\left(\partial_{t} F(x, t)\right)^{2}+\frac{1}{2}\left(\nabla_{\omega} F \cdot \nabla_{\omega} F\right)(x, t)\right] d_{\omega} x \\
& =\int_{\bar{S}}\left[\left(\partial_{t} F(x, t)\right)^{2}+\frac{1}{2} \sum_{y \in \bar{S}}\left(D_{\omega, y} F(x, t)\right)^{2}\right] d_{\omega} x
\end{aligned}
$$

Next theorem shows that the energy of the solutions of the $\omega$-elastic equation are conserved if their boundary values are identically zero.

Theorem 4.6. Let $S$ be a subgraph of a host graph with a weight $\omega$ such that $\partial S \neq \emptyset$. If $F: \bar{S} \times[0, T) \rightarrow \mathbf{R}$ with $F(x, \cdot) \in \mathcal{C}^{1}[0, T) \bigcap \mathcal{D}^{2}(0, T), x \in S$ be a solution of the following $D B V P$

$$
\left\{\begin{array}{l}
\partial_{t}^{2} F(x, t)-\Delta_{\omega} F(x, t)=0, \quad x \in S, t \in(0, T) \\
F(z, t)=0, \quad z \in \partial S, \quad t \in(0, T)
\end{array}\right.
$$

then we have

$$
\boldsymbol{e}(t)=\boldsymbol{e}(0), \quad t \in[0, T)
$$

Proof. It follows from the second assumption in (4.12) that

$$
\partial_{t} F(z, t)=0, \quad z \in \partial S, t \in(0, T),
$$


and

$$
\begin{aligned}
\mathbf{e}^{\prime}(t) & =\int_{\bar{S}}\left[2 \partial_{t} F(x, t) \partial_{t}^{2} F(x, t)+\sum_{y \in \bar{S}} D_{\omega, y} F(x, t) D_{\omega, y} \partial_{t} F(x, t)\right] d_{\omega} x \\
& =\int_{\bar{S}}\left[2 \partial_{t} F(x, t) \partial_{t}^{2} F(x, t)+\left(\nabla_{\omega} F \cdot \nabla_{\omega} \partial_{t} F\right)(x, t)\right] d_{\omega} x \\
& =2 \int_{\bar{S}} \partial_{t} F(x, t)\left(\partial_{t}^{2} F(x, t)-\Delta_{\omega} F(x, t)\right) d_{\omega} x \\
& =2 \int_{S} \partial_{t} F(x, t)\left(\partial_{t}^{2} F(x, t)-\Delta_{\omega} F(x, t)\right) d_{\omega} x=0
\end{aligned}
$$

for all $t \in(0, T)$. Since the function $\mathbf{e}(\mathrm{t})$ is continuous on $[0, T)$, we have the result.

By using the above property of the energy conservation, we can show the uniqueness of the solutions of the DBVP on the $\omega$-elastic equations.

Theorem 4.7. Let $S$ be a subgraph of a host graph with a weight $\omega$ such that $\partial S \neq \emptyset$ and let functions $f, g: S \rightarrow \mathbf{R}, \sigma: \partial S \times(0, T) \rightarrow \mathbf{R}$ and $H: S \times(0, T) \rightarrow \mathbf{R}$ be given. If $F: \bar{S} \times[0, T) \rightarrow \mathbf{R}$ with $F(x, \cdot) \in$ $\mathcal{C}^{1}[0, T) \bigcap \mathcal{D}^{2}(0, T), x \in S$ be a solution of the following $D B V P$

$$
\left\{\begin{array}{l}
\partial_{t}^{2} F(x, t)-\Delta_{\omega} F(x, t)=H(x, t), \quad x \in S, t \in(0, T) \\
F(z, t)=\sigma(z, t), \quad z \in \partial S, \quad t \in[0, T) \\
F(x, 0)=f(x), \quad x \in S \\
\partial_{t} F(x, 0)=g(x), \quad x \in S
\end{array}\right.
$$

then $F$ is uniquely determined.

Proof. Let $F_{1}$ and $F_{2}$ be functions satisfying the hypotheses above and define

$$
H(x, t):=F_{1}(x, t)-F_{2}(x, t), \quad x \in \bar{S}, t \in[0, T) .
$$

Then it is easily seen from Theorem 4.6, that the energy function e corresponding to $H$ is identically zero. Hence, by the definition of $\mathbf{e}$, we have

$$
D_{\omega, y} H(x, t)=(H(y, t)-H(x, t)) \sqrt{\frac{\omega(x, y)}{d_{\omega} x}}=0,
$$

for all $x, y \in \bar{S}, t \in[0, T)$, which implies that $F_{1} \equiv F_{2}$. 


\section{References}

[B] N. Biggs, Algebraic graph theory, Second edition, Cambridge Univ. Press, Cambridge, 1993.

[CB] S.-Y. Chung and C. A. Berenstein, $\omega$-Harmonic functions and inverse conductivity problems on networks, SIAM J. Appl. Math. 65 (2005), no. 4, 1200-1226 (electronic).

[Ch] F. R. K. Chung, Spectral graph theory, Published for the Conference Board of the Mathematical Sciences, Washington, DC, 1997.

[CvDS] D. M. Cvetković, M. Doob and H. Sachs, Spectra of graphs, Academic Press, New York, 1980.

[CY] F. Chung and S.-T. Yau, Discrete Green's functions, J. Combin. Theory Ser. A 91 (2000), no. 1-2, 191-214.

[E] L. C. Evans, Partial differential equations, Amer. Math. Soc., Providence, RI, 1998.

[MC] E. B. Curtis and J. A. Morrow, The Dirichlet to Neumann map for a resistor network, SIAM J. Appl. Math. 51 (1991), no. 4, 1011-1029.

[W] D. V. Widder, The heat equation, Academic Press, New York, 1975. 